\title{
THE NEHARI MANIFOLD FOR A SINGULAR ELLIPTIC EQUATION INVOLVING THE FRACTIONAL LAPLACE OPERATOR
}

\section{ABdelJabBar GHANMi And KAMEL SAOUdi}

Abstract. In this work we study the following singular problem involving the fractional Laplace operator:

$$
\left(\mathrm{P}_{\lambda}\right)\left\{\begin{array}{l}
\mathscr{L} u=\frac{a(x)}{u^{\gamma}}+\lambda f(x, u) \text { in } \Omega ; \\
u=0, \text { in } \mathbb{R}^{N} \backslash \Omega,
\end{array}\right.
$$

where $\Omega \subset \mathbb{R}^{N}, N \geqslant 2$ be a bounded smooth domain, $a \in C(\Omega), \lambda$ is a positive parameter and $0<\gamma<1,2<r<2_{s}^{*}$ where $2_{s}^{*}=\frac{N 2}{N-2 s}$. Under appropriate assumptions on the function $K$ and the function $f$ and we employ the method of Nehari manifold in order to show the existence of $T_{r, \gamma}$ such that for all $\lambda \in\left(0, T_{r, \gamma}\right)$, problem $\left(P_{\lambda}\right)$ has at least two solutions.

Mathematics subject classification (2010): 34B15, 37C25, 35R20.

Keywords and phrases: Fractional Laplace operator, the method of Nehari manifold, singular elliptic equation, multiple positive solutions.

\section{REFERENCES}

[1] C. O. Alves, A. El Hamidi, Nehari manifold and existence of positive solutions to a class of quasilinear problems, Nonlinear Anal. 60 (2005) 611-624.

[2] J. P. Aubin, I. Ekeland, Applied Nonlinear Analysis, Pure Appl. Math., Wiley-Interscience Publications, 1984.

[3] D. Applebaum, Lévy Processes and Stochastic Calculus, seconded., Camb. Stud. Adv. Math., 116, Cambridge University Press, Cambridge, 2009.

[4] B. Barrios, E. Colorado, A. De Pablo, U. Sanchez, On some critical problems for the fractional Laplacian operator, J. Differential Equations 252 (2012), 6133-6162.

[5] K. J. BROWN, Y. ZHANG, The Nehari manifold for a semilinear elliptic equation with a sign-changing weight function, J. Differential Equations 193 (2003) 481-499.

[6] X. CABRÉ, J. TAN, Positive solutions of nonlinear problems involving the square root of the Laplacian, Adv. Math. 224 (2010), 2052-2093.

[7] L. Caffarelli, L. Silvestre, An extension problem related to the fractional Laplacian, Comm. Partial Differential Equations 32 (2007), 1245-1260.

[8] M. M. Coclite And G. Palmieri, On a singular nonlinear Dirichlet problem, Comm. Partial Differential Equations 14 (1989), no. 10, 1315-1327.

[9] H. Chen, H. HAJAIEJ, Y. WANG, On a class of semilinear fractional elliptic equations involving outside Dirac data, Nonlinear Anal. 125 (2015), 639-658.

[10] M. G. Crandall, P. H. Rabinowitz And L. TARTAR, On a Dirichlet problem with a singular nonlinearity, Comm. Partial Differential Equations 2 (1977), no. 2, 193-222.

[11] R. Cont And P. Tankov, Financial Modelling with Jump Processes, Chapman and Hall/CRC Financ. Math. Ser. 2004.

[12] P. DrabeK And S. I. Pohozaev, Positive solutions for the p-Laplacian: application of the fibering method, Proc. Royal Soc. Edinburgh Sect A, 127 (1997) 703-726.

[13] A. Ghanmi, K. SaOUdi, A multiplicity results for a singular problem involving the fractional p-Laplacian operator, Complex variables and elliptic equations 61, 9 (2016) 1199-1216. 
[14] J. GIACOMONI AND K. SAOUdi, Multiplicity of positive solutions for a singular and critical problem, Nonlinear Anal. 71 (2009), no. 9, 4060-4077.

[15] J. GiACOMONI, I. SChindLER AND P. TAKÁČ, Sobolev versus Hölder local minimizers and global multiplicity for a singular and quasilinear equation, annali della scuola normale superiore di pisa, classe di scienze série V, 6 (2007) 117-158.

[16] E. Di. Nezza, G. Palatucci And E. Valdinoci, Hitchhiker's guide to the fractional Sobolev spaces, Bull. Sci. Math. 136 (2012) 521-573.

[17] K. SAOUDI AND M. KRATOU, Existence of multiple solutions for a singular and quasilinear equation, Complex Var. Elliptic Equ. 60 (2015), no. 7, 893-925.

[18] R. Servadei, E. Valdinoci, Mountain Pass solutions for non-local elliptic operators, J. Math. Anal. Appl. 389 (2012), 887-898.

[19] R. SERVADEI, E. VALDINOCI, Variational methods for non-local operators of elliptic type, Discrete Contin. Dyn. Syst. 33 (2013), 2105-2137.

[20] R. SERVADEI AND E. VAlDinOCI, On the spectrum of two different fractional operators, Proc. Roy. Soc. Edinburgh Sect. A 144 (2014), no. 4, 831-855.

[21] J. TAN, The Brézis-Nirenberg type problem involving the square root of the Laplacian, Calc. Var. Partial Differential Equations 36 (2011), 21-41. 